\title{
Detection of Moving Cast Shadow and Removal for Video Surveillance using HSV Color Space
}

\author{
Komal Gogi ${ }^{1}$, Dr. M. Uttarakumari ${ }^{2}$ \\ Communication and System, R.V.College of Engineering and Technology, Bangalore, India ${ }^{1}$ \\ Professor, R.V. College of Engineering and Technology, Bangalore, India ${ }^{2}$
}

\begin{abstract}
Shadow Detection and Removal is the procedure to enhance the execution, reliability and accuracy of the computer vision applications including image segmentation and object recognition, object tracking, reconnaissance and so forth. Shadow area causes false recognition for moving objects. Shadow is also reason for false connectivity and the texture loss of background of independent blobs. We separate the moving object by subtraction algorithm in light of the distinction of pixels. Variance property, Texture and intensity in HSV color space are utilized to distinguish the shadow region and shadow removal is based on the data from the reference frame. Background data is initially stored in reference frame. The next incoming frames with shadow object are compared with this reference frame. The main objective to review this paper is to show better performance.
\end{abstract}

Keywords: shadow detection, shadow removal, subtraction algorithm, HSV color space.

\section{INTRODUCTION}

Shadow is formed when an opaque object barricades the light from its source. A shadow in the computer vision applications has turned into serious problem as it causes various issues as contortion of object shape, object merging, and actual object loss; accordingly shadow produces a negative effect on the accuracy and efficiency of the outcome. In this manner, effective and robust shadow detection techniques are fundamental for the computer vision applications for example surveillance, object recognition, traffic analysis, tracking systems etc. In general, shadows can be separated into two major classes: self and cast shadows. A self-shadow happens in the bit of a question which is not lit up by direct light.A cast shadow is the region anticipated by the object towards direct light. In view of intensity, the shadows are of two types - hard and soft shadows. The soft shadows holds the texture of the background surface, whereas the hard shadows are excessively dull and have little texture.

Thus the detection of hard shadows is complicated as they may be mistaken as dark objects rather than shadows. The detection of a shadow as an authentic moving region may make misperception for the progressive phases of motion analysis and tracking. False edges and boundary of shadows may prompt to slip-ups in visual preparing. The challenges that may merge when shadow pixels are misleadingly ordered as foreground which prompts illusive structures or false connectivity between the object blob and shadow blob. So, the significance of detection of shadow of moving objects has been anextended tradition and in the meantime despite everything it remains a fortifying issue. A shadow appears on a part when the light from a source can't achieve the part because of barricade by an object. We propose a simple technique to detect and remove the shadows from Video frames in RGB color space using HSV color space. In addition, our work represents one of the most elegant approaches to date for detecting both indoor and outdoor shadows and it is consequently capableof working on scenes intruding from different perspectives

\section{METHODOLOGY}

\section{A. Subtraction Algorithm}

The primary target it to distinguish moving object utilizing subtraction algorithm for sequence of frames from video captured. Moving object identification is essential as it contains the shadow with the object. Flowchart of the proposed method for moving object detection is given in Fig. 1. Initially, the video is isolated into frames. An initial frame is taken as a reference frame which contains no moving object in it. The following arriving frames are contrasted with this frame to recognize the moving object. The frame that contains the moving object in it, is called the foreground model. We identify the moving object based on a novel approach that measures the distinction of pixels between the reference frame and the foreground frame. If there is difference between the current frame and the reference frame in pixels then the varied pixels are counted as moving object. In this way, legitimately finding the moving object containing the shadow in beginning stride is the main essential for further shadow detection procedure. The most important condition in our proposed strategy for moving item recognition is the camera ought to be static. 


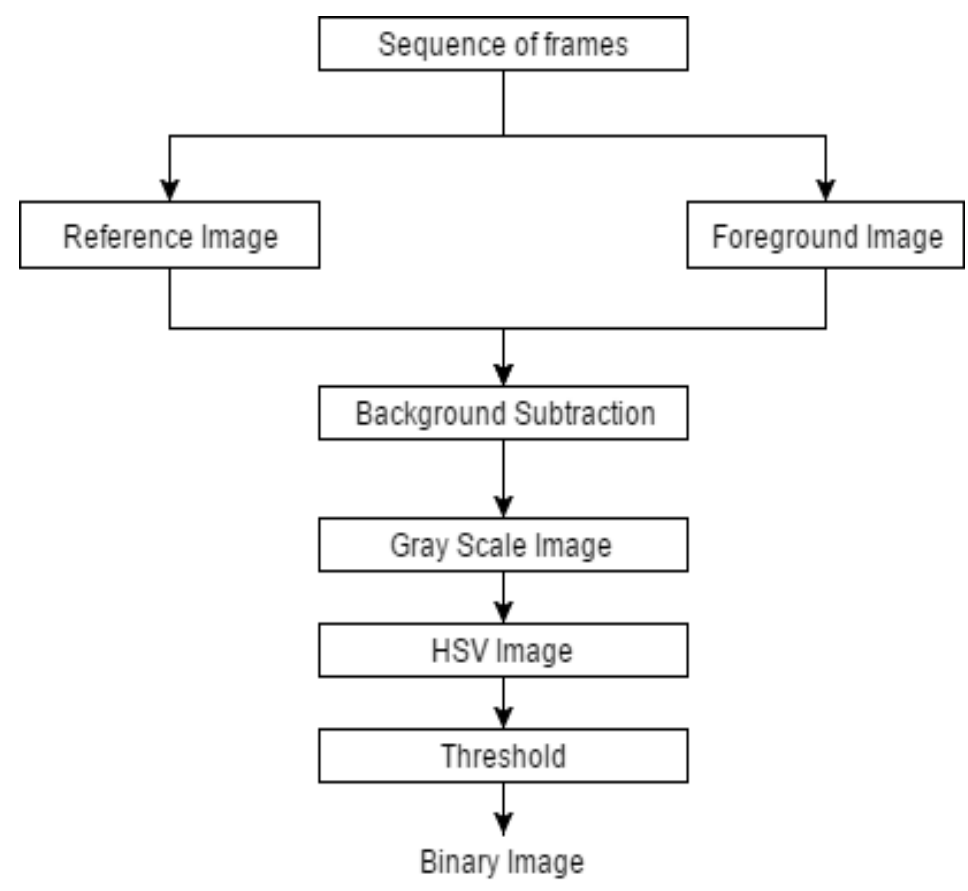

Fig 1. Flowchart of Subtraction Algorithm to Detect Moving Object
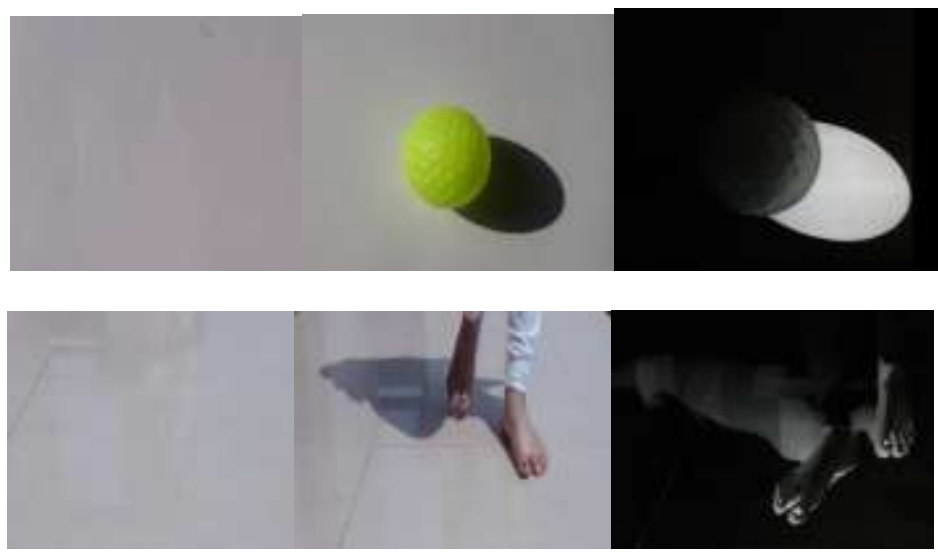

Fig 2. Results of the Flowchart a) Reference image b) Foreground image c) Subtracted image

\section{B. Shadow Detection}

Shadow detection is the principle procedurefor the final image analysis assignment of this paper. The foreground model and the background model from the first segment are utilized forimage division. The foreground model is separated by the background model to deliver the divided image. In case of recognizing the shadow region, the key thought in regards to the divided image is to highlight the homogeneity property of shadow. The proportion between pixels when enlightened and similar pixels can be roughly linear. So, from this property we can measure the abrupt change of the surface in case of texture.

The divided image can be represented as

$$
\text { Divided Image }(x, y)=\frac{\text { Foreground Image }}{\text { Reference Image }}(1)
$$

To take care of stated issues, appropriate detection of the shadows can significantly enhance the result of the image processing. We utilized binary image and the divided image from the preceding section as input in our next section in order to recognize the shadow region. The primary reason for utilizing the binary image is to constrain our operations only into region of the moving object. Presently we figure the mean of every R, G, B channel ofthe divided image. The shadow or the darker locales have less difference in case of color property than the illuminated or colored regions. The estimation of the object blob in each R, G, B channel which are less than the mean of each R, G, B channel are considered for further handling to detect shadow. 


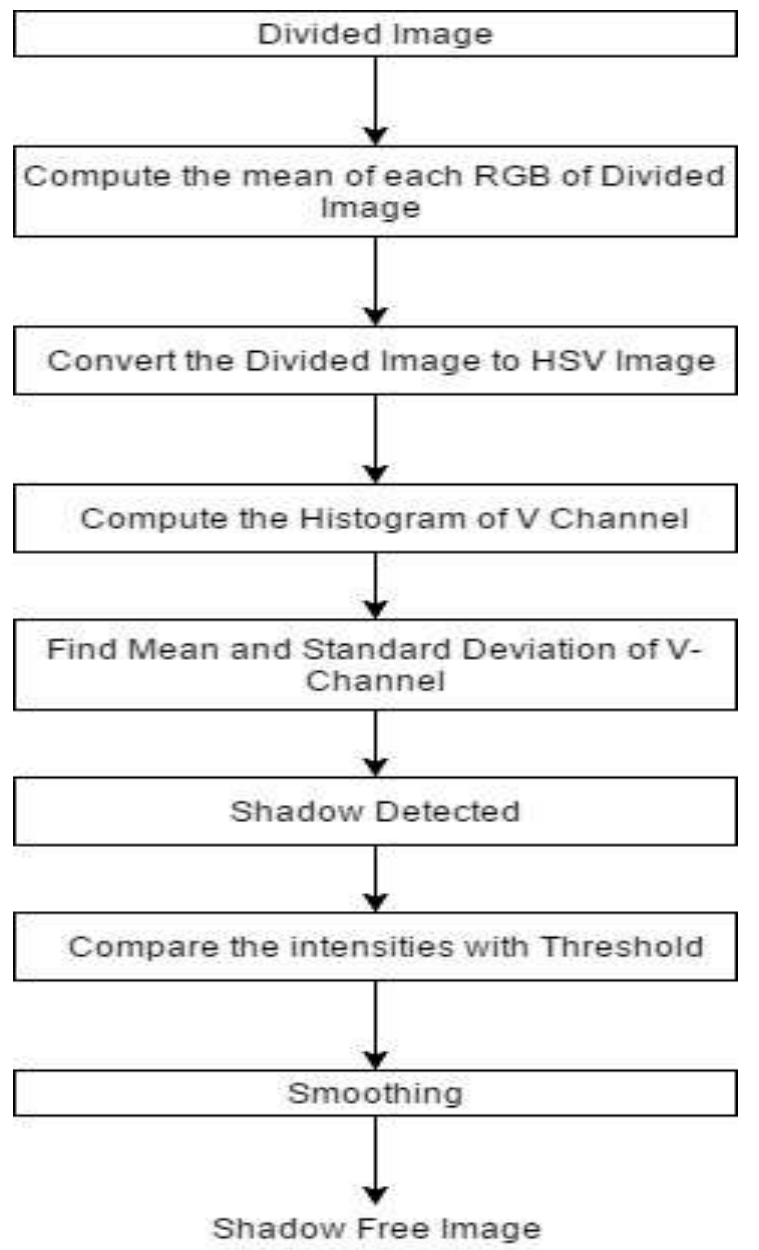

Fig 3. Proposed Method for Shadow Detection and Removal
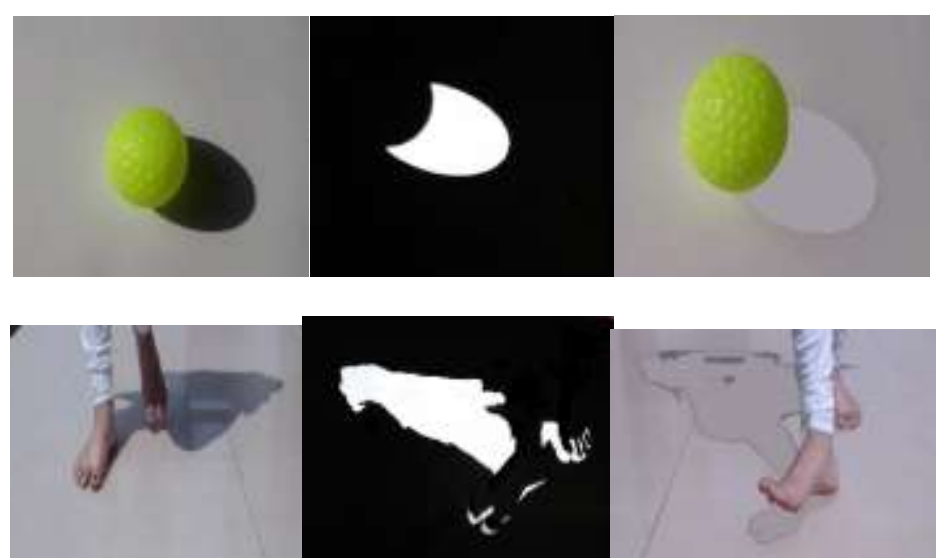

Fig 4. Results for Shadow Detection and Removal Results

In this way, we additionally approach a method based on statistical intensity. We convert the divided image (x, y) into equivalent HSV image. The V channel represents the luma whereas $\mathrm{H}$ and $\mathrm{S}$ represent hue (basic color) and saturation (purity of the color) respectively. Unlike RGB, HSV isolates the image intensity in V channel. Presently aiming at the $\mathrm{V}$ channel, histogram equalization is computed in order to get a more difference image. We process the mean and standard deviation of the image at $\mathrm{V}$ channel. We characterize the threshold value based on statistical approach over a couple tests by applying Gaussian distribution method. With a specific end goal to choose which pixels belong to the shadow region, we compare the pixel intensities against the threshold value. The pixelvalues are taken into account that has the intensity between a ranges that has been selected as threshold value. We consider being part of the shadow, the pixels that have the intensity of $68 \%$ of the first Gaussian distribution. 


\section{Mean}

Mean is most fundamental of all statistical measure. Means are frequently utilized as a part ofgeometry and analysis; an extensive variety of meanshave been developed for these purposes.

In challenge of image processing filtering using mean is classified as spatial filtering and used for noise reduction, works on an sliding ' $\mathrm{m} \times \mathrm{n}$ ' window by calculating the average of all pixel values inside the window and replacing the center pixel value in the destination image with the result. Its mathematical formulation is given as follows

$$
f(x, y)=\frac{1}{(m n)} \sum_{(r, c) \in W} g(r, c)(2)
$$

Where ' $\mathrm{g}$ ' is the noisy image, $\mathrm{f}(\mathrm{x}, \mathrm{y})$ is the restored image, and ' $\mathrm{r}$ ' and ' $\mathrm{c}$ ' are the row and column coordinates respectively, inside a window ' $\mathrm{W}$ ' of size ' $\mathrm{m} \times \mathrm{n}$ ' where the operation takes place. The arithmetic mean filter causes a certain amount of blurring (proportional to the window size) to the image, thereby reducing the effects of noise and local variations.

\section{Standard Deviation}

It is a most generally utilized measure of variability or diversity utilized in statistics. As far as for image processing it indicates how much variation or "dispersion" exists from the average (mean, or expected value).

A low standard deviation demonstrates that the information points tend to be very close to the mean, whereas high standard deviation indicates that the information points are spread out over a large range of values. Mathematically standard deviation is given by:

$$
f(x, y)=\left(\sqrt{\frac{1}{m n} \sum_{(r, c) \in W}\left(g(r, c)-\frac{1}{m n-1} \sum_{(r, c) \in W} g(r, c)\right)}\right)^{2}
$$

A standard deviation channel figures the standard deviation and assigns this value to the center pixel in the yield outline. As it has capability in measuring the fluctuation, it can be utilized as a part of the edge sharpening, as intensity level get changes at the edge of image by vast esteem. Standard deviation channels can be valuable for radar images. The interpretation of radar images is frequently troublesome: you cannot depend on spectral values because of back scatter (return of the pulse sent by the radar). This regularly causes a lot of 'noise'. By utilizing a standard deviation channel, you may be able to recognize some patterns.

\section{Shadow Removal}

Most of the methods did not properly remove the shadows but shadow removal can be a great factor reaching to our desired outcome. Consequently, we propose an adaptable technique that eliminates the shadowappropriately. Our proposed method for shadow removal doesn't just take out the shadow appropriately; it additionally keeps all the desired features intact so the loss of information is reduced. We take the foreground model, binary shadow mask, reference model as inputs for shadow removal.

The principle target of utilizing the binary shadow mask from the previous methodology is to restrict our operations only into region of detected shadow part. As we are working with the background frame and the foreground frame; after detecting the shadow region from the foreground RGB image, we utilized the background frame keeping in mind the end goal to discoverthe region which does not contain the moving object with shadow. We analyzed the foreground model and background model to eliminate the shadow.

The foreground shadow region is recognized first and the pixels in the similar region of the background frame are coincided on the foreground frame. By which, the original texture is gained and we find the moving object that does not contain any shadow attached to it. In this way we got the entire frame that does not contain any shadow and all the background features are additionally perfect.

\section{Discussion}

We have executed the entire method in Visual Studio 2015 with OpenCV open sourcefiles. To affirm the viability of our proposedalgorithm, we took both indoor and outdoor frame sequences as input. We converted the input video into consecutive frames. Our review emphases on RGB frame sequences captured by static camera. We can see that the moving objects for both indoor and outdoor frames are extracted legitimately through our proposed method. Through our proposed method of shadow detection based on statistical intensity we are able to detect the shadow and remove shadow regions for both indoor and outdoor scenes. 

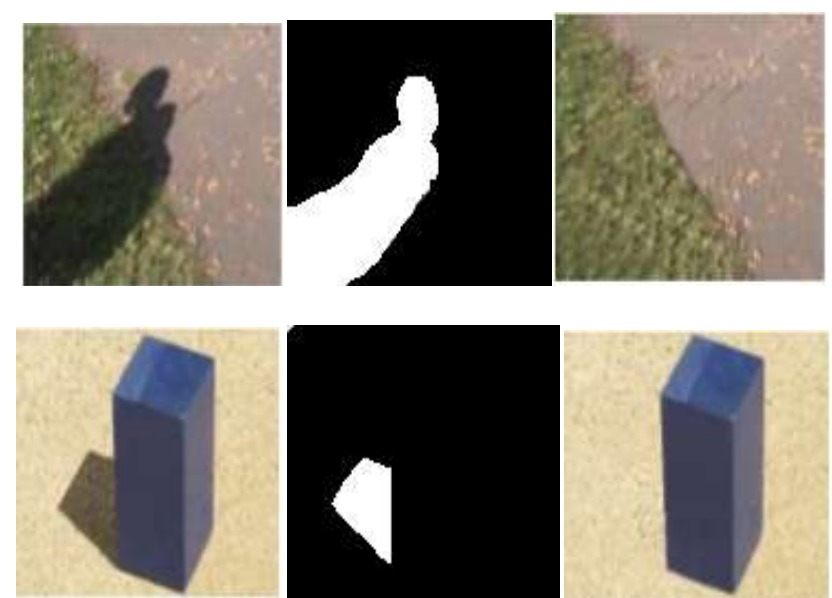

Fig 5. Few examples on proposed Method for Shadow Detection and Shadow Removal

\section{CONCLUSION}

In this paper, we propose aviable background subtraction approach for moving object detection and shadow variance property,suppression based texture of the color and statistics of intensity of pixels in HSV color space. A portion of benefit of our paper are: the texture and the object that have been blocked by the shadow region are totally recovered, presence of object can be detected by detecting the shadow, complex interaction of geometry regarding shadow is detected, tracking of the object may be done by detecting the shadow regions for sequences of frames. Meanwhile, our proposed approach can keep the exhaustiveness of the extracted moving object. Experimental results indicate how the shadow removal and detection module that we set enhance the performance of the overall system. From the results of the experiments, we can also validate that our proposed method is flexible,fast simple, and robust.

\section{REFERENCES}

[1] Kideog Jeong and Christopher Jaynes," Moving Shadow Detection using a Combined Geometric and Color Classification Approach", Proceedings of the IEEE Workshop on Motion and Video Computing 2005.

[2] Manoj K Sabnis, Research Scholar, Dr. Manoj Kumar Shukla, "Shadow Detection of Static Images by InnovativeThresholding Approach", 2011.

[3] Rohini Mahajan and Abhijeet Bajpayee, "An approach for Shadow Detection and Removal based on Multiple Light Sources", May 2015.

[4] Pavan K Shastry and K. R. Ramakrishnan, "Fast Technique for Moving Shadow Detection in Image Sequences", IEEE 2004.

[5] Li-Qun Xu†, José Luis Landabasoł , Montse Pardàs,’SHADOW REMOVAL WITH BLOB-BASED MORPHOLOGICAL RECONSTRUCTION FOR ERROR CORRECTION", 2014

[6] Vijay Chondagar, Harshiv Pandya, Mehul Panchal, Rinkesh Patel, Dhaval Sevak and Kaushal Jani, "A Review: Shadow Detection and Removal”, 2016.

[7] Chuanxu Wang and Weijuan Zhang,"A Robust Algorithm for Shadow Removal of Foreground Detection in Video Surveillance", Asia-Pacific Conference on Information Processing 2009.

[8] Sanjeev Kumar and Anupreet Kaur," ALORITHM FOR SHADOW DETECTION IN REAL COLOUR IMAGES", 2010.

[9] Dr. Sudhir S. Kanade and Mr. Raut Anant S," Detection and Removal of Object-Oriented Shadows from Urban High-Resolution RSI", 2015.

[10] Jyoti Bala1, Ritika2, "Shadowing in Digital Images: Detection \& Removal", International Journal of Advanced Research in Computer Science and Software Engineering, May 2015.

[11] Jing Ji_Xudong Jiang_Wei Sun," Shadowdetection Using Double-Threshold Pulse Coupled Neural, Networks", IEEE 2016.

[12] Henri NICOLAS IRISA/INRIA," Scalable Video Compression Scheme for Tele-Surveillance ApplicationsBased On Cast Shadow Detection and Modelling", IEEE 2005.

[13] Tie-Min Chen, Wei-Xing Wang, "Image Shadow Detection and Classification Based On Rough Sets “, Proceedings of the Fifth International Conference on Machine Learning and Cybernetics, Dalian, 13-16 August 2006.

[14] Yunda Sun Ming Li Wei Wu Baozong Yunn Xiaofang Tang," Background Model Initialization In Moving Object Detection With Shadow Eiimination", IEEE 2004.

[15] Divya K A, Roshna K I and Shelmy Mathai, "Shadow Detection and Removal by Object-wise Segmentation”, IEEE 2015.

[16]Yuqi Wang, Guangxi Zhu, Miao Tang,“An Improvement on Cast Shadow Detection for Vehicles Through Separately Locating Sub Shadow Regions”, IEEE 2006.

[17]Wei Zhang, Xiang Zhong Fang, Yi Xu," Detection of moving cast shadows using image orthogonal transform”, Proceedings of the 18th International Conference on Pattern Recognition 2006.

[18]Wei Zhang, Xiang Zhong Fang, Xiaokang Yang," Moving cast shadows detection based on ratio edge”, IEEE 2006.

[19]Pooya Sarabandi, Fumio Yamazaki, Masashi Matsuoka, Anne Kiremidjian,"Shadow Detection and Radiometric Restoration in Satellite High Resolution Images", IEEE 2004.

[20]Zhiwei Li, Huanfeng Shen, Huifang Li, Liangpei Zhang,"AUTOMATIC CLOUD AND CLOUD SHADOW DETECTION IN GF-1 WFVIMAGERY USING MULTIPLE FEATURES", IEEE 2016.

[21]Kaushik Deb and Animesh Kar, "Moving Cast Shadow Detection and Removal from Video Based on HSV color space", 2015. 